\title{
Preparation of Lyotropic Liquid Crystals and Optical Characterisation
}

\author{
Hiromasa Goto \\ Division of Materials Science, Faculty of Pure and Applied Sciences, \\ University of Tsukuba, Tsukuba, Ibaraki 305-8573, Japan \\ E-mail: gotoh@ims.tsukuba.ac.jp
}

Keywords: ionic liquid, Iyotropic liquid crystal, amphotropic

\begin{abstract}
Synthesis of an ionic liquid crystal (1-(6-(4-(4-trans-pentyl-cyclohexyl)phenoxy)-hexyl)pyridinium chloride) showing lyotropic smectic phase was carried out. A simple reaction between phenylcyclohexane-type liquid crystal having halogen atom in the terminal and pyridine allows production of the ionic liquid crystal. The NMR measurements confirm chemical structure of the compound. Observation with polarsing optical microscopy evaluated that the compound shows fine multi-domains having fluidity. The lyotropic LC thus prepared displays smectic phase with layer structure.
\end{abstract}

\section{Introduction}

Ionic liquids have been paid attention because insolubility in water and some organic solvents. Synthesis of ionic liquid is simple, such as addition of organic molecules with halogen atom to pyridine or imidazole (Scheme 1) [1].

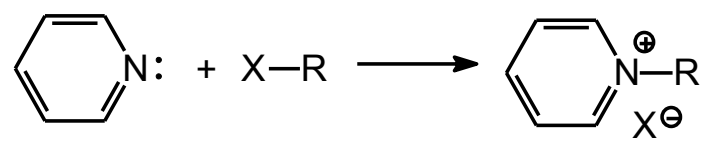

Scheme 1. An example of synthesis of ionic liquid. $X=$ halogen, $R=$ alkyl group.

Liquid crystal is intermediate phase (mesophase) between crystal (solid) and liquid, showing crystal like order and fluidity. Liquid crystallinity involves two categories, such as thermotropic liquid crystallinity (change in "temperature" induces phase transition) and lyotropic liquid crystallinity (liquid crystal phase can form in "solution" at appropriate concentration). In general, lyotropic liquid crystals have low viscosity. Both liquid crystals exhibit birefringence under cross Nicol condition with a polarising optical microscope.

Up to date, many functional ionic liquid crystals have been synthesised [2-7]. Ujiie et al. synthesised thermotropic ionic liquid crystalline polymers in 1991 [8]. First, coupling reaction between 4-hydroxy-2,2,6,6-tetramethyl-piperidine (TEMPOH) as a precursor reaction was attempted in this study to obtain liquid crystal having paramagnetic group, however the reaction afforded a pyridine-based lyotropic liquid crystal. In the evaporation process after the reaction, a residual fluid in the round bottom flask could not be evaporated. Observation with a polarising optical microscopy (POM) found that the liquid shows liquid crystallinity with birefringence. The fluid could not be dissolved in hexane (however, soluble in $\mathrm{CHCl}_{3}$ ). This is a typical character of ionic materials.

The liquid crystal thus synthesised shows smectic phase with good fluidity. In this report, liquid crystal texture of the compound and plausible structure are discussed. 


\section{Experiment}

\subsection{Synthesis}

1-(6-Chloro-hexyloxy)-4-(4-trans-pentyl-cyclohexyl)-benzene, 1.

Sodium (3.56 g, $0.155 \mathrm{~mol}$ ) was slowly added to $100 \mathrm{~mL}$ of ethanol. After sodium was completely dissolved in ethanol due to formation of ethoxide, 4-(trans-4-pentylcyclohexyl)phenol (38.16 g, $0.155 \mathrm{~mol}$ ) was added to the solution and stirred for 1 night at room temperature. Then, 1-bromo-6-chloro hexane $(30.9 \mathrm{~g}, 0.155 \mathrm{~mol})$ was added to the solution and refluxed. After $24 \mathrm{~h}$, the crude product was washed with water, and extracted with ether. The organic layer was washed with $10 \% \mathrm{HCl}$ solution, and collected with separatory funnel. The solution was evaporated followed by recrystallisation from ethanol afforded $33.9 \mathrm{~g}$ of compound $1 \mathrm{Y}=40.0 \%$.

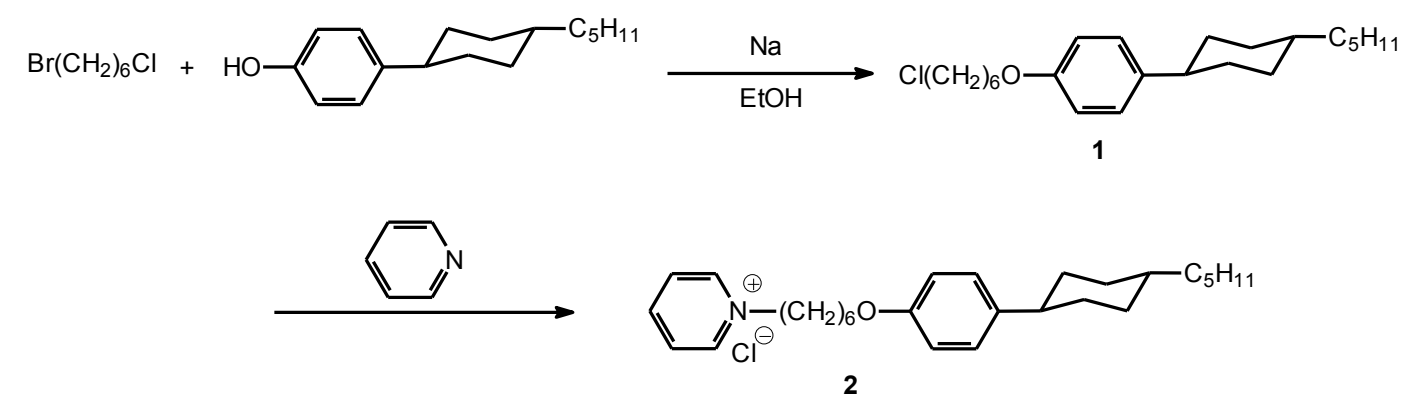

Scheme 2. Synthetic route for compound 2.

1-(6-(4-(4-Trans-pentyl-cyclohexyl)-phenoxy)-hexyl)pyridinium chloride, 2.

Compound $1(10 \mathrm{~g})$ was dissolved in pyridine $(40 \mathrm{~mL})$ and refluxed at $102{ }^{\circ} \mathrm{C}$ in the presence of 4-hydroxy-2,2,6,6-tetramethyl-piperidine (TEMPOH) (TEMPOH was used as the first attempt). After 6 days, the solution was gradually cooled to rt. Evaporation of residual pyridine yielded brown liquid $(5.4 \mathrm{~g})$. In the first attempt, TEMPOH was added in the reaction to obtain compound 3. However, a coupling reaction between compound 1 and TEMPOH was not successfully performed. On the other hand, ionisation reaction between compound 1 and pyridine was progressed to form ionic liquid crystal. Next, the reaction between compound 1 and pyridine in the no presence of TEMPOH could yield compound 2, as well.

\subsection{Characterisation}

Figure 1 shows infra-red (IR) absorption spectrum of compound 1. Absorption bands at 2924, $2853 \mathrm{~cm}^{-1}$ were observed due to $\mathrm{CH}_{2}, \mathrm{CH}$ stretching vibrations. An absorption band due to $\mathrm{C}=\mathrm{C}$ stretching (phenyl) is observable at $1612 \mathrm{~cm}^{-1} \cdot p$-Substituted phenyl group shows an absorption at $1512 \mathrm{~cm}^{-1}$. A stretching vibration due to ether group is observed at $1246 \mathrm{~cm}^{-1}$. Absorptions at 835 and $810 \mathrm{~cm}^{-1}$ are due to $\mathrm{C}-\mathrm{Cl}$ stretching. This result indicates that the compound shows vibrations due to alkyl and aromatic groups. Especially, The IR measurement confirms that the chemical structure of the mesogene having flexible alkyl group with $\mathrm{Cl}$ in the terminal. 


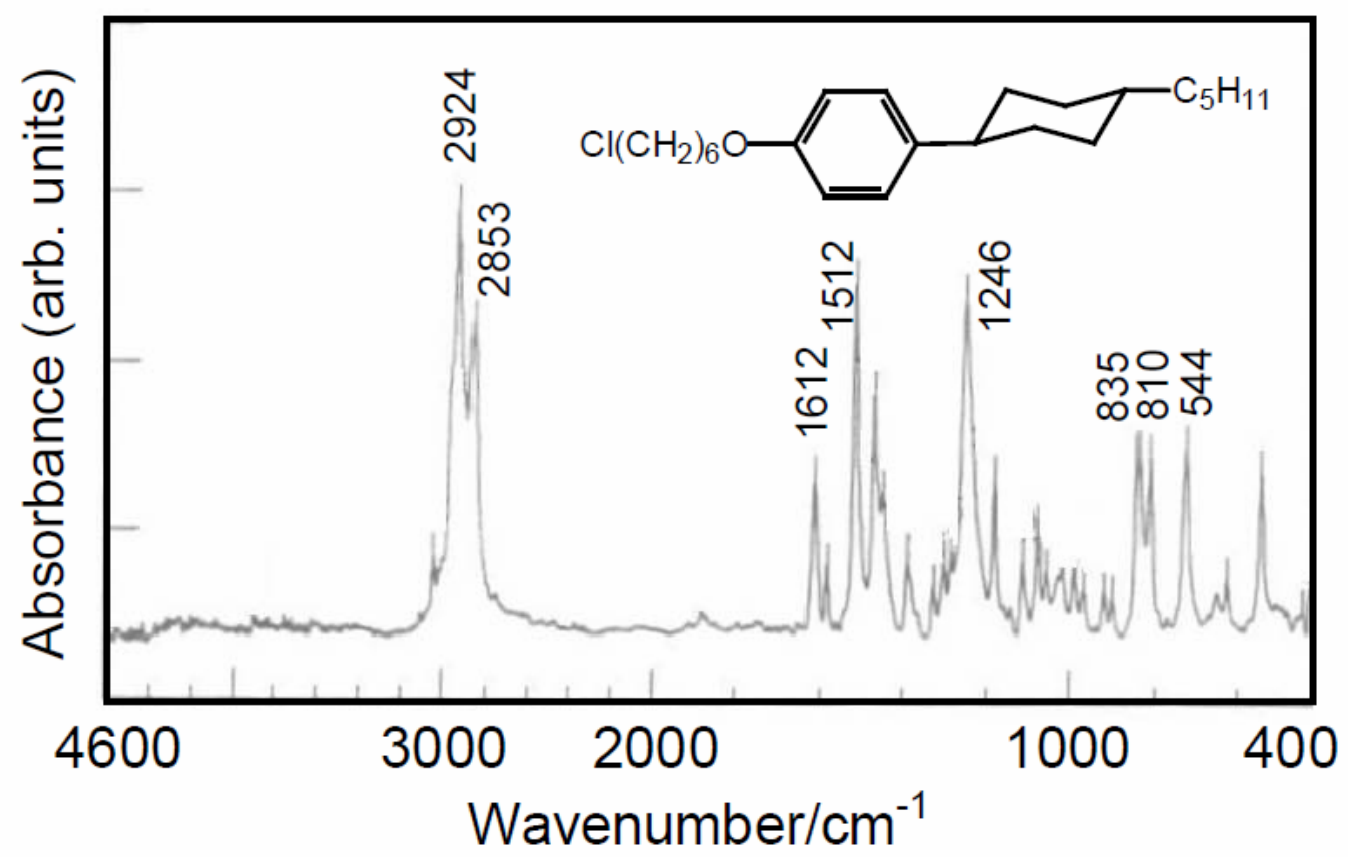

Figure 1. IR absorption spectrum of compound 1.

Aromatic protons of benzene ring in the mesogenic core are observed (two signals, 10,11 in Figure 2). Signals due to protons of terminal pyridine ring are detected at low-magnetic region in the ${ }^{1} \mathrm{H}$ NMR (1-3 signals), Figure 2. ${ }^{13} \mathrm{C}$ NMR and distortion enhancement by polarised transfer (DEPT) spectrum for compound 2 confirm the chemical structure. Aromatic carbons are observed at low-magnetic region (>110 ppm, signals of 1-3 and 10-13), Figure 3a. Here, carbons having even number of hydrogen show downward signals $(14,17,22)$ and odd number upward in the DEPT (Figure 3a).
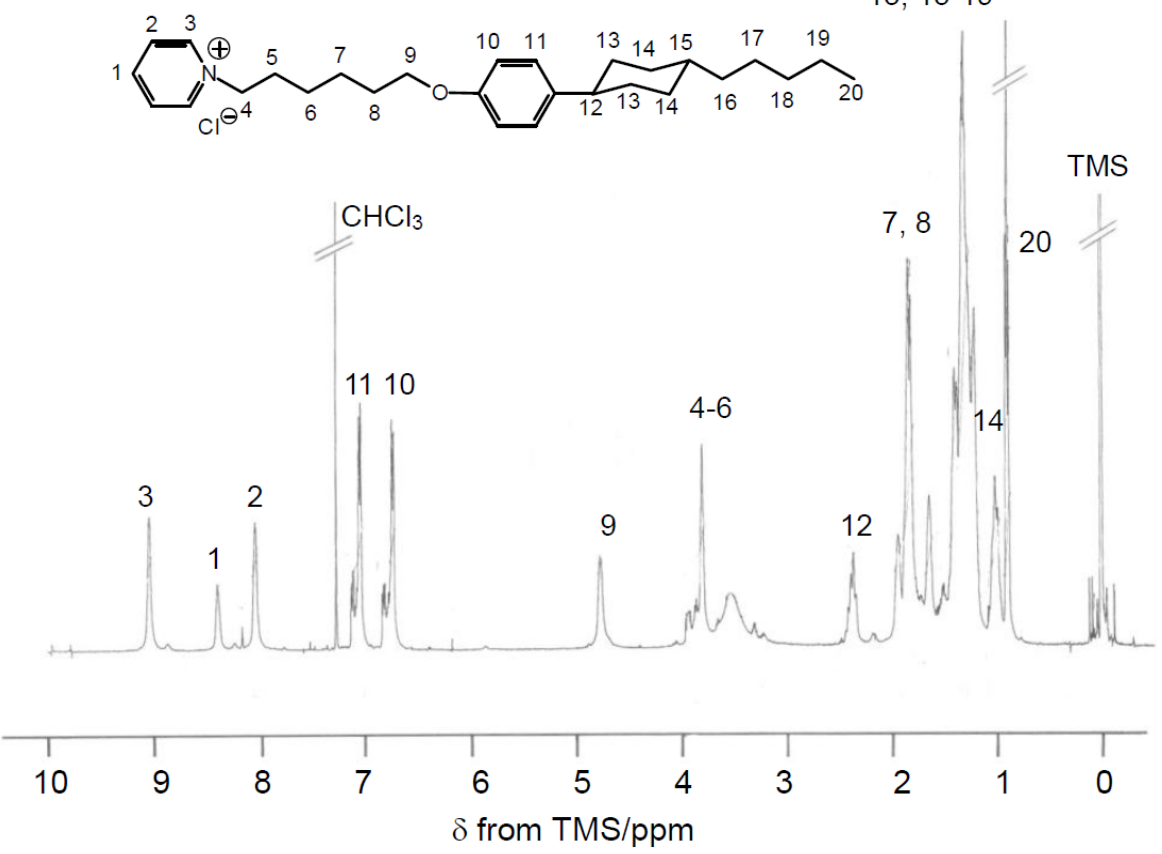

Figure 2. ${ }^{1} \mathrm{H}$ NMR spectrum of compound 2. 
a)
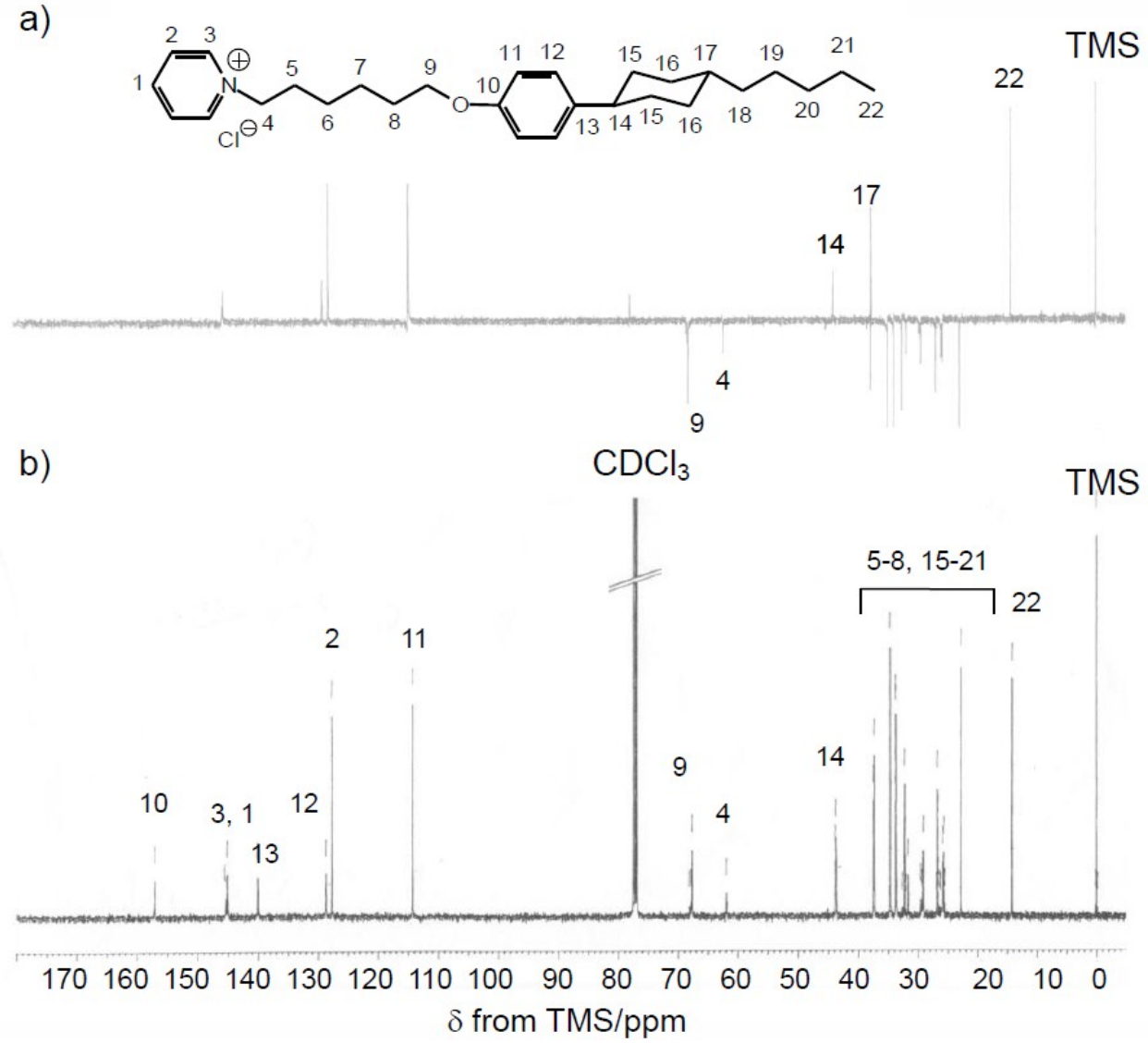

Figure 3. (a): Distortion enhancement by polarised transfer spectrum, (b): ${ }^{13} \mathrm{C}$ NMR.

\subsection{Optical texture}

Polarising optical microscopy (POM) image of compound 2 in pyridine solution showing smectic oily structure is shown in Figure 4(a). Formation of smectic phase indicates the liquid crystal has layer structure. Magnification image of the POM is displayed in Figure 4(b). Thin needle like domain structure was observed, indicating the smectic oily streaks are consisting of the needle like domains. The texture is easily flowed by mechanical force, because of low viscosity derived from the lyotropic liquid crystal character. Note that, compound 1 exhibits thermotropic nematic phase with schlieren texture under observation of the POM, although compound 1 show no lyotropic liquid crystallinity. Compound 2 individually shows mesophase with temperature before phase transition to isotropic phase. Therefore, compound 2 is "amphotropic liquid crystal" having both thermotropic and lyotropic liquid crystallinity. 


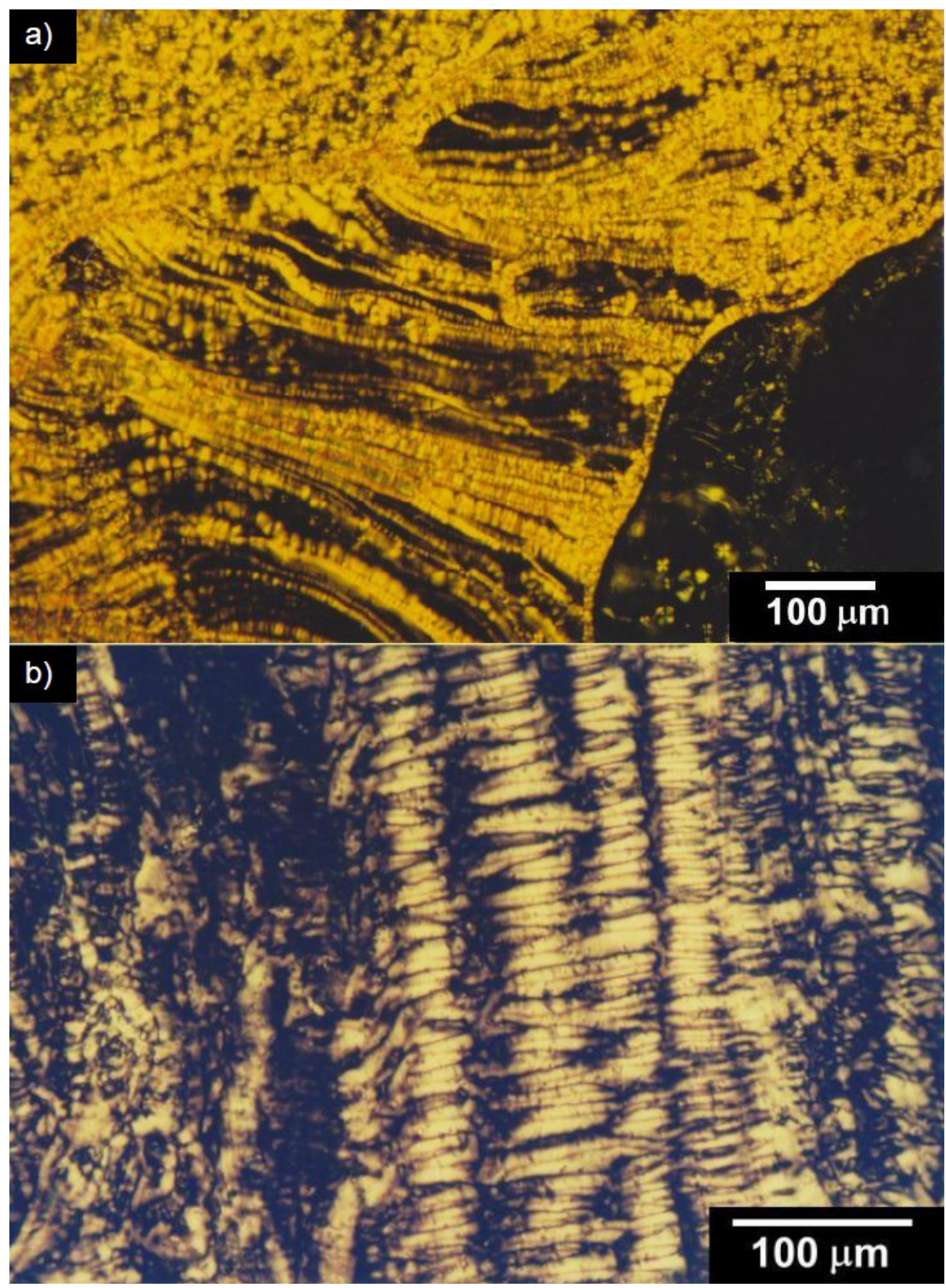

Figure 4. (a): Polarising optical microscopy (POM) image of a smectic oily streak texture of compound 2 at $30^{\circ} \mathrm{C}$. (b): Magnification image of compound 2 observed with the POM. 


\section{Plausible model}

a)

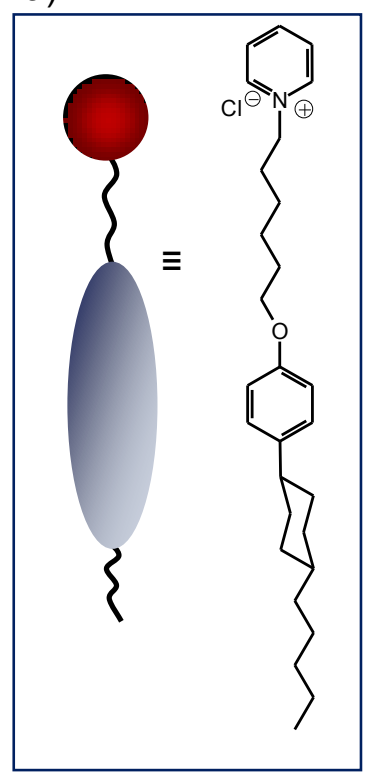

b-1)

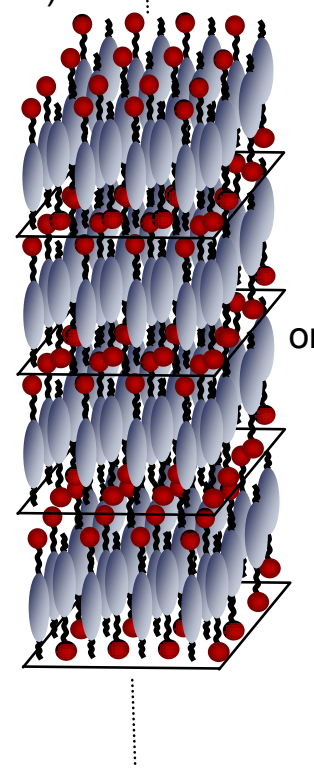

b-2)

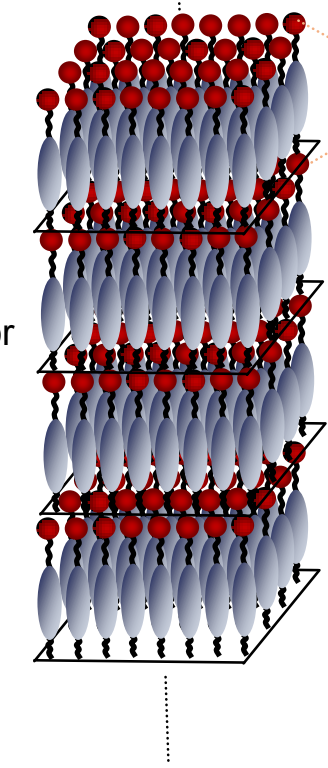

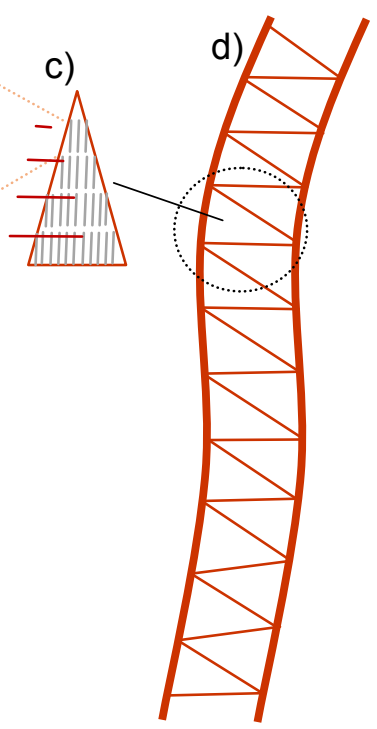

Figure 5. Plausible structure of compound 2 in lyotropic liquid crystal state. (a): Molecular structure and simplification form. (b-1): Interdigitated molecular arrangement to form layer structure of smectic phase. (b-2): Arrangement of molecules in the same direction in the layer structure. (c): Smectic liquid crystal domain structure. (d): Oily streak structure consisting of smectic liquid crystal domains.

Figure 5 displays plausible structure of compound 2 in the lyotropic liquid crystalline state. The previous study proposed interdigitated molecular arrangement to form layer structure of smectic phase [8]. Molecular structure and simplification form are displayed in Figure 5(a). The molecules of compound 2 can form interdigitated arrangement to form layer structure of smectic phase, as shown in Figure 5(b-1), or arrangement of molecules in the same direction in the layer structure (Figure 5(b-2)). The aggregation of layer structure forms smectic liquid crystal domain structure (Figure 5(c)). The smectic domains assemble like a thread to form oily streak structure, as shown in Figure 5(d).

\section{Conclusions}

Ionic-lyotropic liquid crystal showing smectic phase was prepared by a simple reaction. Generally, smectic liquid crystals have high viscosity, while lyotropic smectic liquid crystal thus synthesised show good fluidity. Thermotropic nematic liquid crystals have been applied for displays because of high speed response against electric field, which is derived from their low viscosity. Therefore, ionic-lyotropic liquid crystals with low viscosity have possibility of application for molecular orientation devices showing quick response.

\section{Techniques}

Infrared spectroscopic measurement was carried out with a JASCO FT-IR 300 spectrometer using the KBr method. NMR spectra were obtained with a Bruker AVANCE-500 spectrometer. 
$\mathrm{CDCl}_{3}$ was used as a deuterated solvent, and tetramethylsilane (TMS) was used as an internal standard. Optical textures were observed using a Nikon ECLIPS LV 100 high-resolution polarising microscope with Nikon LU Plan Fluor and Nikon CFIUW lenses.

\section{Acknowledgments}

This work was supported by Chemical Analysis Division Research Facility Center for Science and Technology of University of Tsukuba.

\section{References}

[1] T. Kitazume, M. Kiazume, Wonder of ionic liquid (in Japanese), Kogyo Chosakai Publishing Co., Ltd., 2007, Tokyo. ISBN978-4-7693-4210-6.

[2] S. Ujiie, A. Mori, Cubic mesophase formed by thermotropic liquid crystalline ionic systems effects of polymeric counter ion, Mol. Cryst. Liq. Cryst. 437 (2005) 25-31.

[3] M. Yoshio, T. Kato, T. Mukai, M. Yoshizawa, H. Ohno, Self-assembly of an ionic liquid and a hydroxyl-terminated liquid crystal: Anisotropic ion conduction in layered nanostructures, Mol. Cryst. Liq. Cryst., 413 (2004) 99-108.

[4] J. Wu, S. Ujiie, Ionic liquid crystalline materials exhibiting smectic C phase, Mol. Cryst. Liq. Cryst. 563 (2012) 67-74.

[5] P.K. Son, J.H. Seo, J.C. Kim, T.-H. Yoon, J.H. Park, Ion beam alignment of liquid crystal on amorphous SiOx film, Mol. Cryst. Liq. Cryst. 475 (2007) 65-72.

[6] K. Binnemans, Ionic liquid crystals, Chem. Rev. 105 (2005) 4148-4204.

[7] C. J. D. W. Bruce, Bowlas, K. R. Seddon, Chem. Commun. (1996) 1625-1626.

[8] S. Ujiie, K. Iimura, Thermotropic liquid-crystalline ioninc polymers, Chem. Lett. (1991) 411-414. 